\section{A Novel Design of E-Textile Integration for Physiological Monitoring and Lighting}

Tzu-Hao Huang ${ }^{1 *}$, Xuyuan $\mathrm{TaO}^{2}$, Ya-Chi Ko', Po-Chun $\mathrm{Hsu}^{1}$, Chien-Lung Shen ${ }^{1}$,Fen-Ling Chen ${ }^{1}$, Wei-Chung Wang', GwoTsuen Jou ${ }^{1}$, Vladan Koncar ${ }^{2}$

\begin{abstract}
This research was to focus on integrating printed textile circuit board, FPCB (flexible printed circuit board), and IC Chips into a fabric for physiological monitoring. This cloth can be used to detect physiological signals such as heart rate, ECG, movement, temperature, and respiration. The main disadvantage of traditional smart textiles remains in the bulk volume of the attached rigid hardware equipment, which makes the user uncomfortable. To overcome this problem, the main idea is to minimize the hardware size, make it compatible with the textile structure and integrate the electronics into textiles directly. The prototype was tested in indoor/ outdoor environment. All the physiological data were transmitted into a remote database server and an online monitoring system was used to supervise the physical state of wearer. The main advantage of this design is the new type of conductive junction which is smaller and softer. The size of conductive junction can be tremendously reduced down to $0.5 \mathrm{~mm} * 0.5 \mathrm{~mm}$ compared with the current buckle junction. This novel design of the printed electronics embedded textile technology leads the e-textile physiological monitoring system to a higher tendency of the washable, flexible, stretchable and thin characteristic of the pure textile.
\end{abstract}

\section{Keywords}

E-Textile; Physiological monitoring system; Ecg; Textile circuit

\section{Introduction}

The "Wearable Technology industry", as an important sector of the overall "Smart Textile industry", may be perceived as a newlydefined industrial segment derived from the convergence of many incumbent sectors such as flexible and miniaturized electronics, technical textiles, membranes and barrier insulation, etc. With the development of material science and electronic engineering, wearable textronic devices are tremendously miniaturized and their form moves from bulky and rigid to thin and flexible one. More and more devices become invisible and ubiquitous for endusers. The user-machine interfaces become more friendly [1-4] and the wireless systems are extensively involved in the transmission of vital signs monitoring, motion, etc. [5,6].

However, the electronic components are always an obstacle in

*Corresponding author: Tzu-Hao Huang, Taiwan Textile Research Institute, No.6, Chengtian Rd., Tucheng Dist, New Taipei City 23674, Taiwan, E-mail: d95522013@gmail.com

Received: December 04, 2017 Accepted: February 27, 2018 Published: March 02, 2018 terms of application, the connection of electronics and textile are not robust enough, and thus wearable textiles are not ready for the market. Therefore, the purpose of the research was to propose a new way to embed printed electronic circuit into textile. This kind of e-textile can be used to detect physiological signals like heart rate, ECG, movement, temperature, and respiration or lighting. The two crucial components of this novel design are conductive fabric circuit and connection by ACF (Anisotropic Conductive Film). Through the proposed method, the new design leads the e-textile system to a higher tendency of the washable, flexible, stretchable and thin characteristic of the pure textile.

\section{Method}

This study proposed four kind methods to combine the textile and electronic component, shown in Figure 1. There are two main structures, textile circuit and electronic component. The textile circuit can be constructed by $1 \mathrm{~A}$ : printing circuit on textile with TPU (Thermoplastic Urethane) 1B: knitted, woven, or embroidering circuit on textile directly. The structure of electronic component can be constructed by 2A: IC chips on FPCB or 2B: Only IC chips. Then there are four kinds of methods to combine them, M1: 1A2A, M2:1A2B, M3:1B2A, M4:1B2B. Due to the ACF excellent property (The ACF insulates in horizontal direction and conducts in perpendicular to plane direction, it is chosen to connect the textile circuit and electronic component. Because the ACF is usually used in semiconductor industry such as touch panel, and it was thought that it might be the first time to be used in the textile industry. The parameters for manufacturing and their washability need to be looked into. Four sections will be discussed: (1) the parameter test for the connection between textile circuit and electronics; (2) the washability test of the textile circuit and the connection ; (3)the method to construct the smart textiles of physiological monitoring, and; (4) the method to construct the LED embedded textiles.

\section{The washability test for the textile circuit}

In order to test the washability of the textile circuit, totally 100 samples were made (10 widths $(0.2 \mathrm{~mm}, 0.3 \mathrm{~mm}, 0.4 \mathrm{~mm}, 0.5 \mathrm{~mm}$, $0.75 \mathrm{~mm}, 1 \mathrm{~mm}, 2 \mathrm{~mm}, 3 \mathrm{~mm}, 4 \mathrm{~mm}, 5 \mathrm{~mm}) \times 10$ samples), shown in Figure 2. The samples were printed conductive ink (CI-1036, Engineered Materials Systems, USA) on the textile with TPU (ST-

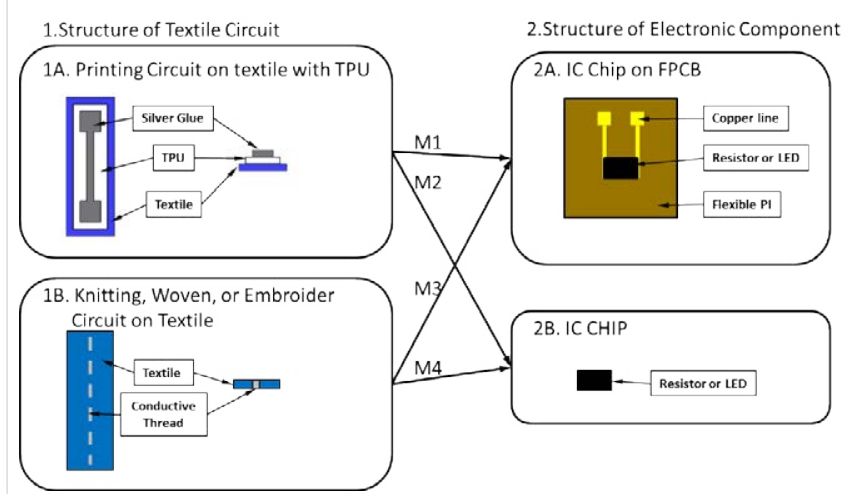

Figure 1: The proposed methods for combination of the textile and electronic component. 
604, BEMIS TAIWAN, Taipei City). Those samples were covered by the TPU and only the resistance measurement pad was exposed. To investigate the washability, the ISO 6330 standard was applied to the washability test [7]. The temperature of $30^{\circ} \mathrm{C}$ was used and 10 wash cycles were run. Every wash cycle was programmed for $30 \mathrm{~min}$. The rotation speed was $30 \mathrm{rpm}$. Between cycles, samples were drip dried in a ventilated oven and their resistance was measured. The washing test machine is Datacolor AHIBA IR.

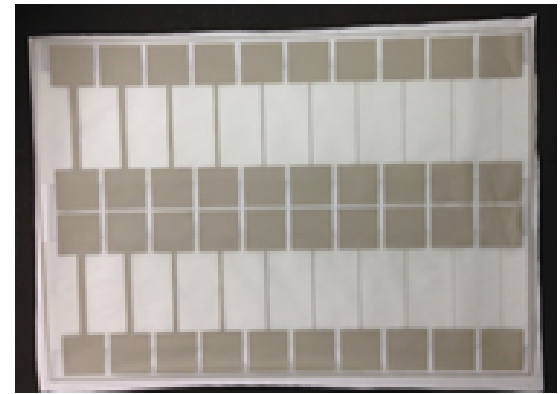

Figure 2: The samples for the printed conductive ink on the textile with TPU.

\section{Smart textile for physiological monitoring}

To integrate the textile and electronics of smart textile for physiological monitoring, the proposed method M1 was applied here. The conductive ink, FPCB (Flexible Printed Circuit Board), and ACF were three key parts in this prototype, shown in Figure 3. First, the particular designed pattern of physiological monitoring electrodes is developed and directly screen printed on textile at a low temperature by conductive ink as the conductive fabric electrodes. Second, FPCB was substituted for RPCB which delivers and calculates physiological signals, and FPCB was pliable enough to be embedded in textile at a low temperature without any uncomfortable sensing and irritation.

Thus, by these 2 special design, the fabric electrodes \& controller's plastic hard shell is likely to vanish from E-textile. Third, the printed electrodes and FPCB were combined by a special heat pressing and glued-laminated procedure, and due to the specific binder, ACF, the junctions between electrodes and FPCB only conduct in z-direction (perpendicular direction of e-textile), shown in Figure 4.

\section{LED embedded textile}

This LED embedded was made by the proposed method M2,

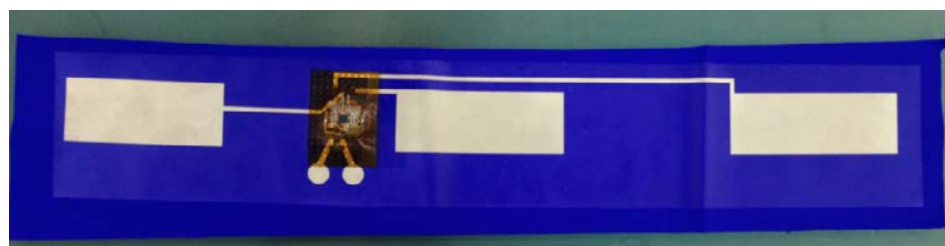

(a) screen printed electrodes

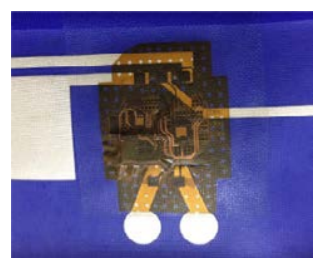

(b) FPCB layer

Figure 3: The elastic conductive ink pattern and FPCB layout on blue textile.

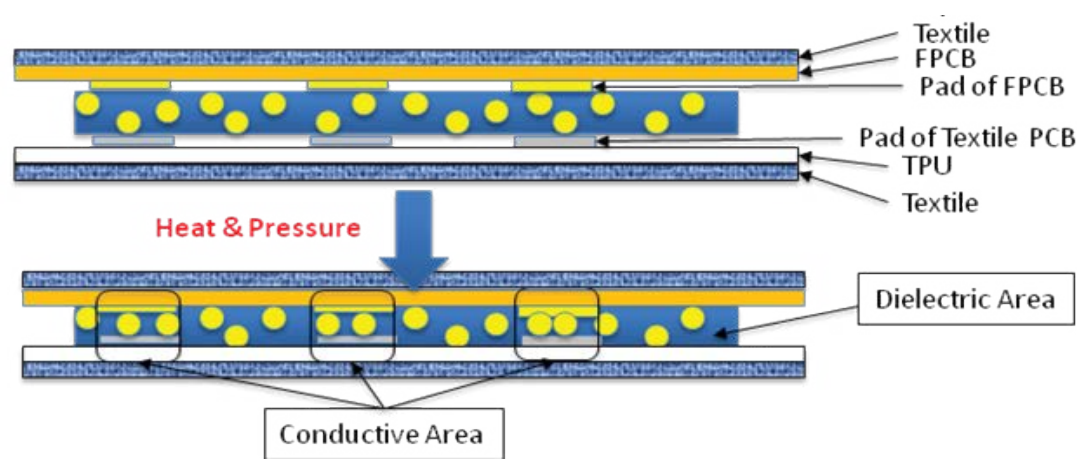

Figure 4: The ACF insulates in x-direction and y-direction (horizontal direction of textile), and only conduct in z-direction (perpendicular direction of textile)

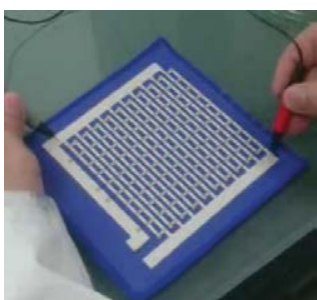

(a) The printed textile circuit and the mounted LEDs

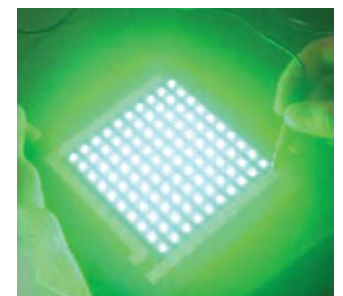

(b) The lighting test for the LED embedded textile

Figure 5: The textile circuit for LED embedded. 


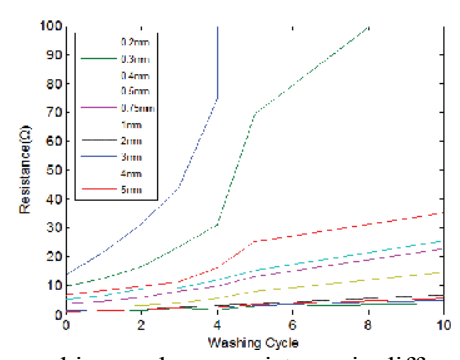

(a) the washing cycles vs resistance in different line widths

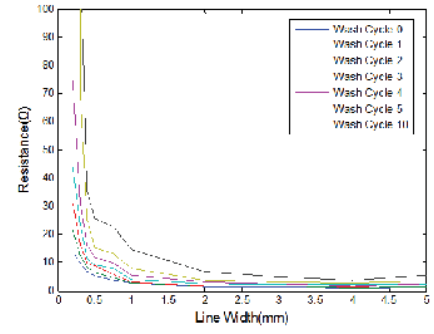

(b) line widths vs the resistance in different washing cycles

Figure 6: The results of the washability test for the textile circuit.

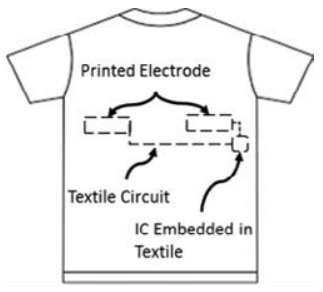

(a) The design concept

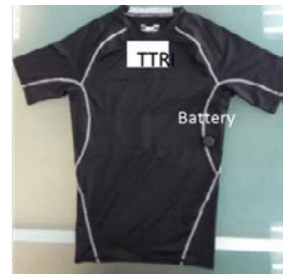

(b) The smart cloth

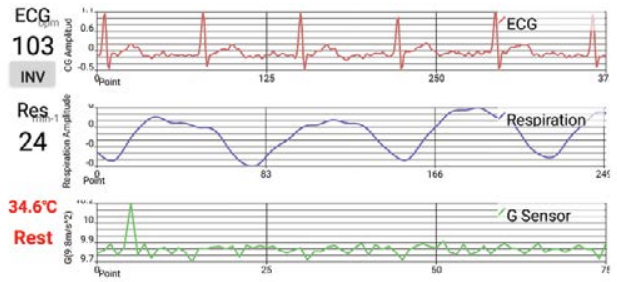

(c) The real-time display in the cell phone

Figure 7: The physiological signals detected by the new design e-textile.

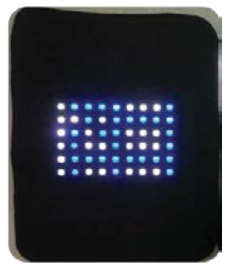

(a) LED textile

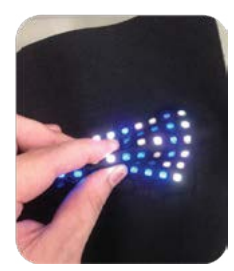

(b) Flexibility demostration (c) LED cloth

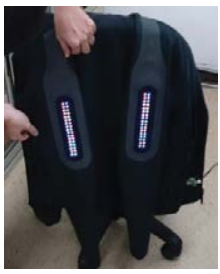

Figure 8: The LED embedded textile and cloth.

which integrated printed circuit board and LEDs into a fabric directly. The LEDs circuit board were made by printing the elastic conductive ink on TPU and the LEDs are glued with the junction on the textile by ACF directly, shown in Figure 5. The main advantages of this design were that the new type of conductive junction was smaller and softer and the LEDs modules was easy to integrate into the textiles by the process of heat and pressure.

The Controllable LEDs Textiles can display any kind of pattern and change the pattern by cell phone application in real time. In the future, it can be used in safety and fashion garment. This novel design of the LEDs embedded textile technology create a new way to achieve the better properties of washability and flexibility for smart textile area.

\section{Result and Discussion}

\section{The results of the washability test for the textile circuit}

The resistance of different widths (the length is $6 \mathrm{~cm}$ and the thickness is $15 \mathrm{um}$ ) are shown as Figure 6. The median resistance of $0.4 \mathrm{~mm}$ is 35.23 after 10 washing cycles. We concluded the width can be used in the textile circuit for the LEDs and physiology sensing as the width is large than $0.4 \mathrm{~mm}$. The 0.2 and 0.3 width line might be too fine to resist the force from washing test.

\section{The prototype of smart textile for physiological monitoring}

The E-textile was softer and washable and all electronic elements were embedded in textiles, shown in Figure 7(a) and Figure 7(b) respectively. The detected physiological signals show in Figure 7 (c). The ECG, heart rate, respiration signal, respiration rate, root-meansquare value of accelerometer, and temperature were measured by the smart cloth and display on the cell phone in real time.

\section{The prototype of LED embedded textile and cloth}

There are two kinds of prototype are made for the demonstration of LED embedded textiles.

Figure $8(a)$ shows the LED textile and the LEDs are single color (white or blue), it only needs the power line and Figure 8(b) demonstrates the flexibility of the LEDs textile. Figure 8(c) shows the LED cloth, the LEDs are RGB LEDs, and those can be controlled by cell phones.

\section{Conclusion}

A novel printed electronic method to create physiological monitoring E-textile and LED embedded textile was proposed in this research. The conductive ink, FPCB, and ACF were used to substitute for fabric electrodes, monitoring controller and buckle interface. Experiment results verified that the proposed method could produce identical physiological signals with traditional E-textile in a more comfortable, softer and lighter way.

\section{Acknowledgement}

This project has received funding partially from the Ministry of Economy 
Citation: Tzu-Hao H, Tao X, Ko YC, Po-Chun H, Shen CL, et al. (2018) A Novel Design of E-Textile Integration for Physiological Monitoring and Lighting. J Fashion Technol Textile Eng S4:010.

doi:10.4172/2329-9568.S4-010

Affairs of the R.O.C. under grants 106-EC-17-A-22-0331(Significant Technology Development Program of Advanced Functional Fibers and Textiles(2/4)) and the European Union's Horizon 2020 research and innovation program under the Marie Skłodowska-Curie grant agreement No.644268.

\section{References}

1. Eichinger GF, Baumann K, Martin T, Jones M (2007) Using a PCB layout tool to create embroidered circuits.

2. Linz T, Gourmelon L, Langereis G (2007) Contactless EMG sensors embroidered onto textile.

3. Chan M, Estève D, Fourniols JY, Escriba C, Campo E (2012) Smart wearable systems: Current status and future challenges, Artif Intell Med 56: 137-156.

4. Stoppa M, Chiolerio A (2014) Wearable electronics and smart textiles: A critical review. Sensors 14: 11957-11992.

5. JaeHan C, Kim Y, Lee K, Chung YC (2008) Various wearable embroidery RFID tag antenna using electro-thread.

6. Linz T, Kallmayer C, Aschenbrenner R, Reichl H (2005) Embroidering electrical interconnects with conductive yarn for the integration of flexible electronic modules into fabric.

7. ISO (2012) Textiles-Domestic Washing and Drying Procedures for Textile Testing.

\section{Author Affiliations}

${ }^{1}$ Taiwan Textile Research Institute, No.6, Chengtian Rd., Tucheng Dist, New Taipei City 23674, Taiwan

${ }^{2}$ Ecole Nationale Supérieure des Arts et Industries Textiles, Roubaix, France

Submit your next manuscript and get advantages of SciTechnol submissions

* 80 Journals

* 21 Day rapid review process

* 3000 Editorial team

* 5 Million readers

* More than 5000

* Quality and quick review processing through Editorial Manager System

Submit your next manuscript at • www.scitechnol.com/submission 